\title{
Denis Diderot, die Schrift der Vorsehung und der aktive Leser
}

Wir könnten ebenso Jean-Jacques Rousseau wie auch Denis Diderot in einen philosophisch-literarischen Zusammenhang stellen mit dem absichtsvollen Versuch, in ihren literarischen oder philosophischen Schriften die göttlichen Instanzen des Lesens und des Schreibens zu desakralisieren und an ihre Stelle menschliche Institutionen oder gar menschliche Figuren treten zu lassen. Im Verlauf des letzten Drittels des 18. Jahrhunderts avancierte die Geschichte als Kollektivsingular ${ }^{1}$ zur großen, in gewisser Weise gottähnlichen Instanz, welche vergleichbar mit dem Jüngsten Gericht ihre Urteile über die Menschheit fällt. Wir haben bei unserer Beschäftigung mit Les Confessions von Rousseau bemerkt, wie an die Stelle des göttlichen Lesers des Lebensbuches, in diesem Falle der Autobiographie, nun der menschliche Leser tritt und das Lesen selbst zur Grundlage jeglichen Erkenntnis- und Sinnbildungsprozesses wird. Und wir können nun mit Denis Diderot erkennen, dass es zwar noch immer ,da oben' eine Vorsehung gibt und eine quasi göttliche Instanz, dass ansonsten aber alles längst irdisch geworden ist und auf Erden verhandelt wird. Die göttlichen Instanzen und ihre jeweiligen weltlichen Vertreter sind ihrer Legitimität und Autorität größtenteils verlustig gegangen. dies blieb nicht ohne Konsequenzen für die Rolle des Lesepublikums.

Einer jener Denker der französischen Aufklärung, die sich dieser Herausforderung vielleicht am entschlossensten und zugleich auch am widerspruchsvollsten gestellt haben, ist der eigentliche Vater der berühmten französischen Encyclopédie, der französische Philosoph Denis Diderot, der von 1713 bis 1784 lebte. Beschäftigen wir uns nur ganz kurz mit einigen biographischen Tatsachen, die uns zum Schaffen dieses außergewöhnlich produktiven Denkers führen sollen!

Der am 5. Oktober 1713 geborene Denis Diderot erhielt von 1723 bis 1732 den ihn prägenden Unterricht am Jesuitenkolleg von Langres, später in Paris. Er versucht in der Folge erfolgreich, sich innerhalb einer sich im vollen Wandlungsprozess der Öffentlichkeit befindlichen französischen Gesellschaft zu behaupten und hält sich mit Theaterrezensionen, Übersetzungen und verschiedensten Texttypen über Wasser. Er nutzt die Zeit, um sich mit so unterschiedlichen Gegenständen wie der Mathematik sowie der griechischen Antike zu beschäftigen, und weitet seinen geistigen Horizont entschlossen aus. Berühmt ist seine eher kurzlebige

1 Vgl. hierzu Koselleck, Reinhart: Vergangene Zukunft. Zur Semantik geschichtlicher Zeiten. Frankfurt am Main: Suhrkamp 1979.

Ә Open Access. (C) 2020 Ottmar Ette, publiziert von De Gruyter. (c) BY-NC-ND Dieses Werk ist lizenziert unter der Creative Commons Attribution-NonCommercial-NoDerivatives 4.0 International Lizenz. https://doi.org/10.1515/9783110665093-014 
Freundschaft mit Rousseau, die im August 1742 begann. Noch waren die beiden Seiten der französischen Aufklärung vereint.

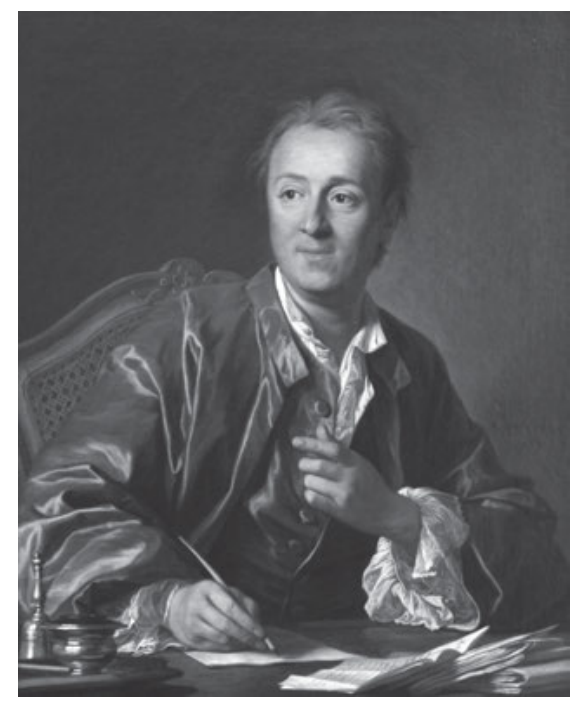

Abb. 79: Denis Diderot

(Langres, 1713 - Paris, 1784).

Im Jahre 1746 veröffentlicht Diderot anonym die Pensées philosophiques, die sofort vom Parlement de Paris dem Feuer überantwortet werden; fortan wird ihr Autor von der Polizei überwacht. Schon im Jahre 1747 aber wird die Grundlage für seine große philosophische Tat gelegt: D’Alembert und er unterschreiben den Gründungsvertrag für eine gemeinsam erstellte Encyclopédie. Neben seiner unermüdlichen Arbeit an diesem Grundlagenwerk beschäftigt er sich immer wieder mit literarischen Spielen oder Fingerübungen und veröffentlicht etwa im Jahr 1748 anonym ein Glanzstück der erotischen Literatur, Les bijoux indiscrets, auf die ich leider in dieser Vorlesung nicht eingehen kann. Der Tod seiner achtundzwanzigjährigen Schwester Angélique als wahnsinnig gewordene Ursulinerin im Kloster setzt ihm zu; er wird 1760 seine vehemente Schrift La Religieuse veröffentlichen.

Seine Lettre sur les Aveugles 1749 bedeuten für sein Denken einen Meilenstein; doch wird er am 24. Juli desselben Jahres per lettre de cachet gesucht, verhaftet und im Donjon von Vincennes eingesperrt. Er nutzt die Zeit zur Übersetzung von Platon, liest die ersten Bände der Histoire naturelle von Buffon und wird am 3. November wieder freigelassen. Und Diderot lässt sich nicht weichkochen. 1750 erscheint sein berühmter Prospectus für die Encyclopédie, das große Gemeinschaftswerk der französischen Aufklärung, deren erster Band im Juni 1751 publiziert und bald schon verboten wird. 
Vergleichbar mit einem Voltaire wird Diderot zu einem Fuchs im Verlagsgeschäft, im Umgehen von weltlichen wie kirchlichen Veröffentlichungsverboten oder im verbotenen Drucken aufklärerischer Schriften unter falschen Verlagsorten. Er versteht es, an den Fäden einer gesamteuropäisch ausgerichteten Verlagslandschaft $\mathrm{zu}$ zupfen. So wird er rasch $\mathrm{zu}$ einem wendigen Intellektuellen avant la lettre. Zielstrebig arbeitet er weiter an den Bänden seiner Encyclopédie, veröffentlicht aber regelmäßig eigene philosophische Schriften, die seinen Ruf als einer der führenden europäischen Denker stärken. Spätestens 1757 ist die Freundschaft zwischen Diderot und Rousseau Geschichte; ab diesem Zeitpunkt lässt sich mit guten Gründen von einer Zweigleisigkeit der französischen Lumières sprechen und von einer Entwicklung in der République des Lettres, welche das kommende Jahrhundert mit der aufkommenden Empfindsamkeit vorbereiten wird.

Von September bis November 1759 verfasst er den Salon de 1759 für die Correspondance littéraire, die auch alle nachfolgenden Salons des Philosophen aufnehmen wird. Diese Schriften sind von grundlegender Bedeutung für die ästhetischen Begriffe und Entwicklungen in der zweiten Hälfte des 18. Jahrhunderts. Diderot ist im Zentrum des französischen Denkens angekommen und darf fortan als einer seiner herausragenden Köpfe gelten. Im Januar 1766 erscheinen die letzten zehn Textbände der Encyclopédie; die Bände mit den berühmten Planches, den Illustrationen und Abbildungen, werden noch bis 1772 nach und nach veröffentlicht.

1772 entsteht auch die erste Fassung seines Supplément au voyage de Bougainville; Diderots Mitarbeit an Guillaume-Thomas Raynals Histoire des deux Indes, einer Art Kolonialenzyklopädie der europäischen Expansion, bleibt den Zeitgenossen zwar zunächst weitgehend verborgen, profiliert ihn aber auch auf diesem Gebiet als einen der maßgeblichen Denker seiner Zeit. Wir haben in unserer Vorlesung bereits untersucht, wie einflussreich seine Rolle als Koautor war und wie wichtig gerade auch seine Wendungen an die Leserschaft für diese Histoire wurden.

Die Zusammenarbeit mit Raynal an diesem Bestseller der Lumières setzt sich bis ins Jahr 1780 fort; 1781 verteidigt er den von Verfolgung bedrohten südfranzösischen Kleriker in seiner polemischen Lettre apologétique de l'abbé Raynal. Die Reisen nach Den Haag und nach St. Petersburg in den Jahren 1773 und 1774 festigen seine Stellung als europäischer philosophe von Rang; doch seine Rückkehr nach Paris führt ihn gleichsam in sein angestammtes geistiges Biotop zurück.

Im November des Jahres 1778 beginnt Diderot mit der Veröffentlichung von Jacques le fataliste et son maître - eine erste Fassung dieses Textes legte er schon 1771 vor - in der Correspondance littéraire von Grimm. Bis zu seinem Tod am 21. Juli 1784 bleibt Diderot einer der produktivsten Philosophen seiner Epoche. 
Ich möchte Ihnen in der Folge einen kurzen Auszug aus dem Kontext der Reiseliteratur präsentieren und dabei zu einer hermeneutischen Grundfigur des Verstehens gelangen, dem Springen. ${ }^{2}$ Diese zweifellos am ,modernsten' anmutende Bewegungsfigur, die exemplarisch vorgestellt und diskutiert werden soll, ist auf den ersten Blick von etwas diffuser Natur und gar nicht so leicht nachvollziehbar. Denn sie betrifft einen Reisebericht und ein dazu gehöriges Verstehensmodell, in welchem weder ein konkreter Ausgangspunkt noch ein konkreter Zielpunkt der Reise angegeben werden. Haben wir es aber dann noch wirklich mit einer realen Reise und einer klaren Verstehensbewegung zu tun? Und wie lassen sich diese Bewegungen dann überhaupt lesen?

Nur auf den ersten Blick scheint es nicht einfach, für das 18. oder 19. Jahrhundert Beispiele für derartige Reisebewegungen anzuführen. Ich möchte daher auf ein der fiktionalen Erzählliteratur entstammendes Beispiel, den erwähnten und von Diderot verfassten Roman Jacques le fataliste et son maître zurückgreifen, der uns noch abschließend etwas über die Bewegungen der Lektüre und des Lesens im Jahrhundert der Aufklärung vermitteln soll. In der berühmt gewordenen Eingangsszene, in welcher der Erzähler mit dem/der Leser`in ein fiktives Zwiegespräch über die Protagonisten dieses Textes führt, treten die spezifischen Grundstrukturen der Reise- und Verstehensbewegungen im Roman deutlich vor Augen:

Wie hatten sie sich getroffen? Durch Zufall, wie jedermann. Wie nannten sie sich? Was kümmert sie das? Woher kamen sie? Vom nächstgelegenen Ort. Wohin gingen sie? Weiß man denn, wohin man geht? Was sagten sie? Der Herr sagte nichts, und Jacques sagte, dass sein Hauptmann sagte, dass alles, was uns hier unten an Gutem und an Schlechtem zustößt, dort oben geschrieben ward.

Der Herr: Das ist ein großes Wort!

Jacques: Mein Hauptmann fügte hinzu, dass für jede Kugel, die aus einem Gewehr abgeschossen wird, die Adresse feststeht.

Der Herr: Und er hatte recht ....

Die intertextuelle Vernetzung mit anderen literarischen Bezugstexten ist schon in dieser Passage komplexer als dies auf den ersten Blick scheinen will. Die referierte Antwort des Capitaine findet sich bereits in Sternes Tristram Shandy: Diderot war ein guter Leser, wobei er bisweilen auch ein guter Plünderer war, der

2 Vgl. zu diesen hermeneutischen Bewegungsfiguren, auf die an dieser Stelle nicht mehr eingegangen werden kann, den vorangegangenen Band der Vorlesungen von Ette, Ottmar: ReiseSchreiben. Potsdamer Vorlesungen zur Reiseliteratur. Berlin - Boston: Walter de Gruyter 2020.

3 Diderot, Denis / Lecontre, Simone (Hg.) / Le Galliot, Jean (Hg.): Jacques le fataliste et son maître. Genève: Droz 1977, S. 3. 
die Texte anderer für seine Zwecke einzusetzen wusste. Dies haben wir schon bei seiner Mitarbeit an Raynals Histoire des deux Indes deutlich vermerkt.

Aber Jacques ist von Tristram Shandy ja gar nicht allzu weit entfernt! Nicht weiter jedenfalls als das Lesen vom Schreiben, denn beide sind nicht nur einander entgegengesetzt, sondern auch komplementär zueinander und vor allem aufeinander abgestimmt. So war auch Hans Magnus Enzensberger ein ausgezeichneter Leser Diderots. Entsprechend heißt es in seinem Radio-Roman Jakob und sein Herr frei nach dem französischen Philosophen:

Hörer: Wie waren die beiden zueinander gekommen?

Erzähler: Von ungefähr, durch Zufall - wie denn sonst?

Hörer: Wo kamen sie her?

Erzähler: Aus dem nächstbesten Dorf an ihrem Weg.

Hörer: Wo gingen sie hin?

Erzähler: Weiß man je, wohin man geht?

Hörer: Wie hießen die beiden?

Erzähler: Sie hießen Jakob und sein Herr.

Hörer: was sprachen die beiden miteinander?

Erzähler: Der Herr kein Wort; Jakob hingegen:

Jakob: Mein Hauptmann, Gott hab ihn selig, hat immer gesagt: Alles, was uns an Gutem oder Bösem auf Erden begegnet, steht dort oben geschrieben.

Herr: Das ist ein großes Wort.

Jakob: Und er pflegte hinzuzusetzen: Jede Kugel, die abgeschossen wird, hat ihre Adresse.

Herr: Dein Hauptmann hatte recht. ${ }^{4}$

Sie sehen durch einen kurzen Textvergleich, dass wir es hier zugleich mit dem literarischen Verhältnis des Lesens als Schreiben, der Übersetzung als Kreation und der intermedialen Transposition - vom Roman zum Radio-Roman - zu tun haben. So verschieben sich die Grenzen zwischen den verschiedenen Modi der Lektüren deutlich und werden im Enzensberger'schen Text selbst undeutlich. Hans Magnus Enzensberger ist hier Leser, Übersetzer und Schriftsteller, er ist also - nach einer lange tradierten philologischen Terminologie - zweifellos zugleich scriptor, compilator und auctor sowie lector in einem. Der Ort „dort oben“ aber ist in Wahrheit längst nicht mehr besetzt: Die Menschen haben die Rolle des göttlichen Lesers wie des göttlichen Schreibers übernommen. Das Lesen ist längst zu einem textproduktiven Vorgang geworden.

4 Enzensberger, Hans Magnus: Jakob und sein Herr. Hörspiel nach dem Roman von Denis Diderot. Rottenburg am Neckar: Diderot Verlag 2006. Erstsendung: 25.12 .1979 (Saarländischer Rundfunk / Südwestfunk). 
Doch bohren wir noch ein wenig weiter. Die Erwartungsklischees der fiktiven Leserfigur werden ein um's andere Mal enttäuscht, ihre Fragen führen nur zu Gegenfragen: Est-ce que l'on sait où l'on va? Der Zufall erscheint von Beginn an als eigentlicher Motor des Geschehens, und doch ist es, wie Erich Köhler in einer denkwürdigen Studie herausarbeiten konnte, ein Zufall, der im dialektischen Spiel mit der geschichtlichen Notwendigkeit ein in der jeweiligen historischen Situation angelegtes Mögliches entfaltet. ${ }^{5}$ Es ist diese Kombinatorik, welche den jeweiligen Sinn hervorbringt, und nicht die Deutung durch einen göttlichen Leser, der aus dem Text eine feststehende Wahrheit herauszieht und verkündet.

Die Lektüre bringt folglich eine je nach historischem Kontext und vielen zusätzlichen Faktoren immer wieder neue ,Wahrheit', einen sich immer wieder verändernden Sinn hervor, den die Leserinnen und Leser stets von neuem erzeugen und für sich gewinnen. Dem Geschehen liegt ebenso wenig ein göttlicher Heilsplan zugrunde wie der Reise ein detaillierter oder auch nur (voraus-) bestimmbarer Fahrplan. Herkunft und Zielort entziehen sich der Kenntnis des Lesers. Ja mehr noch: Dieses Lesepublikum wird am Ende des Romans vom Erzähler aufgefordert, einen Schluss für die Geschichte zu finden und als Leserin - wie wir heute sagen würden - interaktiv zu sein. Zu diesem Zwecke bietet Denis Diderot mit Hilfe seines Erzählers dem Lesepublikum verschiedene Möglichkeiten an, unter denen zu wählen ist. Die Lektüre wird innerhalb einer Verstehensbewegung des Hin- und Herspringens jenseits aller genau festgelegten Reiserouten textproduktiv.

Dies ist ein im Grunde unerhörter Vorgang, welcher der Leserin und dem Leser eine hochaktive Rolle zuweist. Ein genau bestimmbarer Ort der Ankunft wird durch die Betonung einer radikalen Offenheit der Zukunft und des weiteren Reisewegs ersetzt: Weiß man denn, wohin die Reise geht? Ist dem Menschen die Verfügbarkeit über Anfangs- und Endpunkt seines irdischen Lebensweges entzogen, ist ihm also der bewusste und reflektierte Zugang zum Augenblick seiner Geburt - deren Spuren der Körper tragen kann, ohne dass dies doch mehr als eben Spuren wären - wie zum Augenblick seines Todes verwehrt, so bietet ihm der Roman kompensatorisch die Verfügbarkeit über ein gesamtes Leben, über vollständige Lebensläufe an. Die aufmerksame Leserin, der aufmerksame Leser braucht nur zu wählen und dadurch einen immer wieder neuen Sinn der Lektüre, Sinn durch Lektüre zu generieren. Vorbei also die Zeit, in welcher der Sinn für alle Zeiten und für alle Seiten feststand.

5 Vgl. Köhler, Erich: Der literarische Zufall, das Mögliche und die Notwendigkeit. München: Fink 1973, sowie ders.: „Est-ce que l'on sait où l'on va?“ - Zur strukturellen Einheit von „Jacques le Fataliste et son Maître“. In (ders.): Vermittlungen. Romanistische Beiträge zu einer historischsoziologischen Literaturwissenschaft. München: Fink 1976, S. 219-239. 
Durch Abgeschlossenheit wird Sinn erzeugt, im französischen sens ist diese Gerichtetheit des Sinnes mitenthalten. Dieser Zugriff auf eine Totalität an Leben und Lebenserfahrung aber wird der/dem fiktiven Leser`in in der Eingangspassage von Diderots Roman gerade verwehrt, um diese Leserfigur aus der Reserve $\mathrm{zu}$ locken. Diese gezielte Ent-Täuschung prägt die gesamte Handlungsstruktur des Romans, der sich an seinem ,Ende' auf verschiedene, teilweise intertextuell zurückverweisende Varianten öffnet. Gefragt ist nun die/der aktive, die den Text hinterfragende und frei gestaltende Leser ${ }^{\star}$ in.

Jacques le fataliste et son maitre fehlt es ausgehend von seiner Figurenkonstellation nicht an literarischen Bezugstexten. Don Quijotes Reiseweg und die experimentelle Romanform von Sternes Tristram Shandy führen das itinerarische Schema mit seinen Digressionen, Exkursionen und Unterbrechungen zugleich vor und ad absurdum. Der Roman entzieht sich ostentativ der Verfügungsgewalt ebenso eines sinngebenden Autors wie eines göttlichen Lesers. Doch ist gerade das Lesen sein zentrales Moment und Motiv. Diderots kluges literarisches Spiel, seine Fingerübungen für den Roman der Zukunft, weisen den Weg in eine Emanzipation des Lesers und der Lektüre. Aber die Wege der Literaturen der Welt sind oft auch Umwege, welche das Leben einholt. Und das 19. Jahrhundert ging diesen einen Weg, der von Diderot zumindest skizziert worden war, noch nicht entschlossen weiter. Erst im 20. Jahrhundert werden wir wieder auf literarische Texte treffen, welche die von ihm intendierte ,Deregulierung von Schreiben und Lesen in die Tat umsetzen sollten. 